\section{JTI}

JOURNAL OF

TRAUMA AND INJURY

\title{
Heterotopic Ossification in the Abdominal Wall after Exploratory Laparotomy
}

\author{
Hohyun Kim, M.D. ${ }^{1,2}$ \\ ${ }^{1}$ Department of Trauma Surgery, Pusan National University Hospital, Busan, Korea \\ ${ }^{2}$ Biomedical Research Institute, Pusan National University Hospital, Busan, Korea
}

Received: October 17, 2018

Accepted: October 31, 2018

\section{Correspondence to}

Hohyun Kim, M.D.

Department of Trauma Surgery, Biomedical Research Institute,

Pusan National University Hospital,

179 Gudeok-ro, Seo-gu, Busan 49241, Korea

Tel: $+82-51-240-7369$

Fax: +82-51-240-7719

E-mail: gskhh@naver.com
Heterotopic bone formation in abdominal incisions is a recognized but uncommon sequela of abdominal surgery. On the other hand, the formation of ectopic bone is a well-recognized complication following arthroplasty of the hip. Heterotopic ossification of midline abdominal incision scars is a subtype of myositis ossificans traumatica. Ectopic bone formation of midline abdominal incisions may cause regional pain or discomfort in the patient after surgery. If symptomatic, treatment is complete excision with primary closure. Radiologically, it is important to distinguish this benign entity from postoperative complications. We report a 69 -year-old male who underwent exploratory laparotomy for traumatic small bowel perforation. A segment of abnormal hard tissue was found in the abdominal wall. Heterotopic ossification may occur at various sites and is a recognized but infrequent sequela of exploratory laparotomy. This case highlights clinical and etiological features of this finding.

Keywords: Abdominal wall; Heterotopic ossification; Laparotomy; Abdominal injuries

\section{INTRODUCTION}

Heterotopic ossification ( $\mathrm{HO}$ ) refers to the formation of mature lamellar bone at extra-skeletal sites. HO occurs when bone develops in tissues which usually don't undergo ossification. $\mathrm{HO}$ in the abdominal wall, also known as myositis ossificans traumatica, is a rare and benign form of ossification, usually related with previous abdominal surgery or trauma [1].

Since HO was first reported in 1901 by Askanazy and Lubarsh [2], the literature is largely limited to case reports. $\mathrm{HO}$ is considered an uncommon complication following abdominal surgery. However, its true incidence remains unknown given the

This is an Open Access article distributed under the terms of the Creative Commons Attribution Non-Commercial License (http://creativecommons.org/licenses/by-nc/4.0/) which permits unrestricted noncommercial use, distribution, and reproduction in any medium, provided the original work is properly cited. 

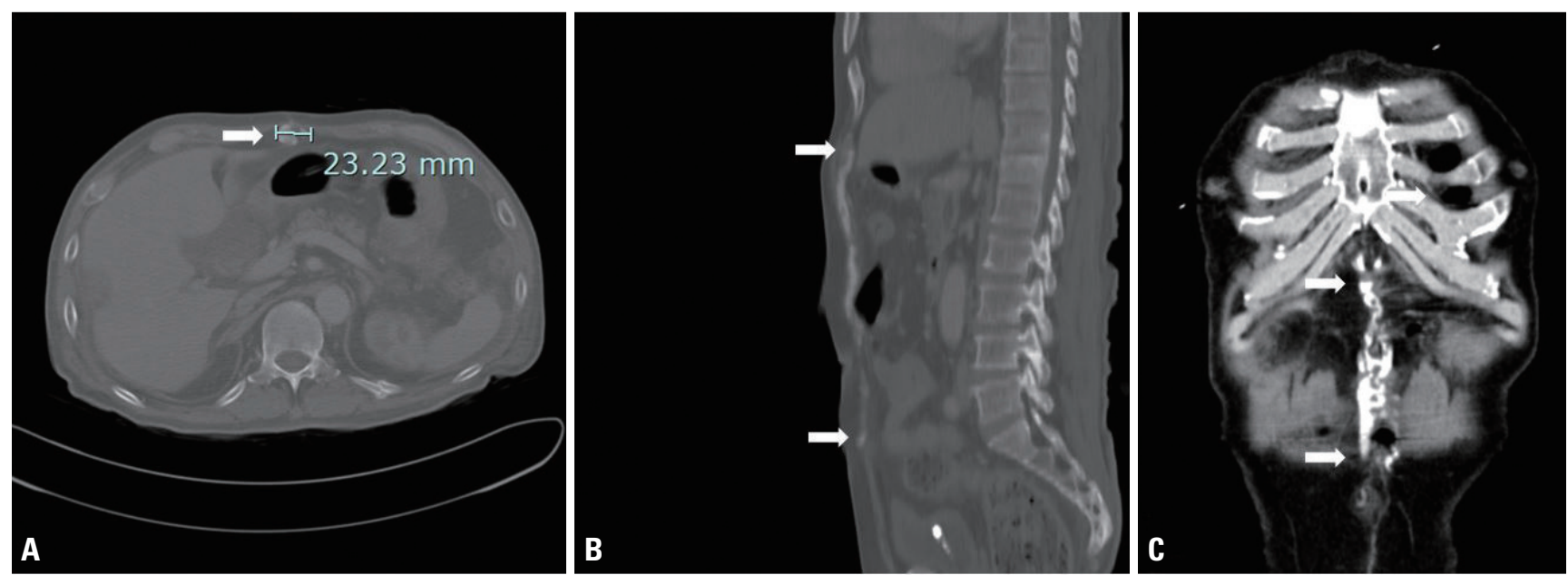

Fig. 1. Abdominal $\mathrm{CT}$ on postoperative 45th day shows heterotopic ossification in the anterior abdominal wall (A), extending from the immediate subxiphoid region ( $\mathrm{B}, \mathrm{C}$; top arrows) to the umbilical region ( $\mathrm{B}, \mathrm{C}$; bottom arrows). The diameter of heterotopic bone was $2.3 \mathrm{~cm}$ ( $\mathrm{A}$, arrow). CT: computed tomography.

scarcity of published reports. The pathogenesis remains incompletely understood. A combination of local injury and systemic stress is thought to create a pro-inflammatory environment, which stimulates heterotopic bone formation [3]. Herein, we report a case of heterotopic ossification in the abdominal wall caused by the exploratory laparotomy with a brief review of the literature.

\section{CASE REPORT}

A 69-year-old male, with no significant past medical history, was involved in a high-speed, road traffic accident. He arrived to the trauma emergency room at our hospital in a stable condition. He was assessed along the lines of the Advanced Trauma Life Support protocol. The Glasgow coma scale was 15 at presentation and the vital signs were within normal limits. The focused assessment with sonography in trauma examination was positive. A contrast-enhanced computed tomography (CECT) scan of the abdomen and pelvis revealed a free peritoneal air and a wall defect and edema of the small intestine. At exploratory laparotomy, approximately $500 \mathrm{~mL}$ of intraperitoneal blood were found. Mesenteric avulsions of the small bowel and sigmoid colon with wide serosal disruption were also founded, requiring resection and anastomosis. The patient recovered after prolonged hospitaliza- tion due to rehab treatment and pressure sore on left heel and was discharged on the postoperative day 31 without clinical evidence of complications.

On postoperative 45 th day, he presented to the outpatient clinic without any clinical symptoms. A healed vertical midline incision in the upper abdomen was found during a physical examination. Physical examination revealed a large, solid, longitudinal structure, in line and directly under the scar of the upper abdomen laparotomy. It was easily palpated and the caudal margin could be held by the examining physician just above the umbilicus. During examination it appeared to move and/ or articulate just below the xyphoid process. Computed tomography (CT) of the abdomen revealed a vertically oriented, linear, calcified lesion in the incision scar on the anterior abdominal wall (Fig. 1A, arrow), extending from the immediate subxiphoid region (Fig. 1B, C; top arrow) to the umbilical region (Fig. 1B, C; bottom arrow). The size of heterotopic bone was $15 \times 2.3 \mathrm{~cm}$. This finding was consistent with heterotopic ossification, a subtype of myositis ossificans traumatica. In this case, no further action was taken; however, the patient was advised to return for a follow-up visit.

During a follow-up visit, he complained an intermittent abdominal wall discomfort, but didn't notice mechanical obstruction of movement and pain. On postoperative 2 years, follow-up CECT scan of the abdomen demonstrat- 

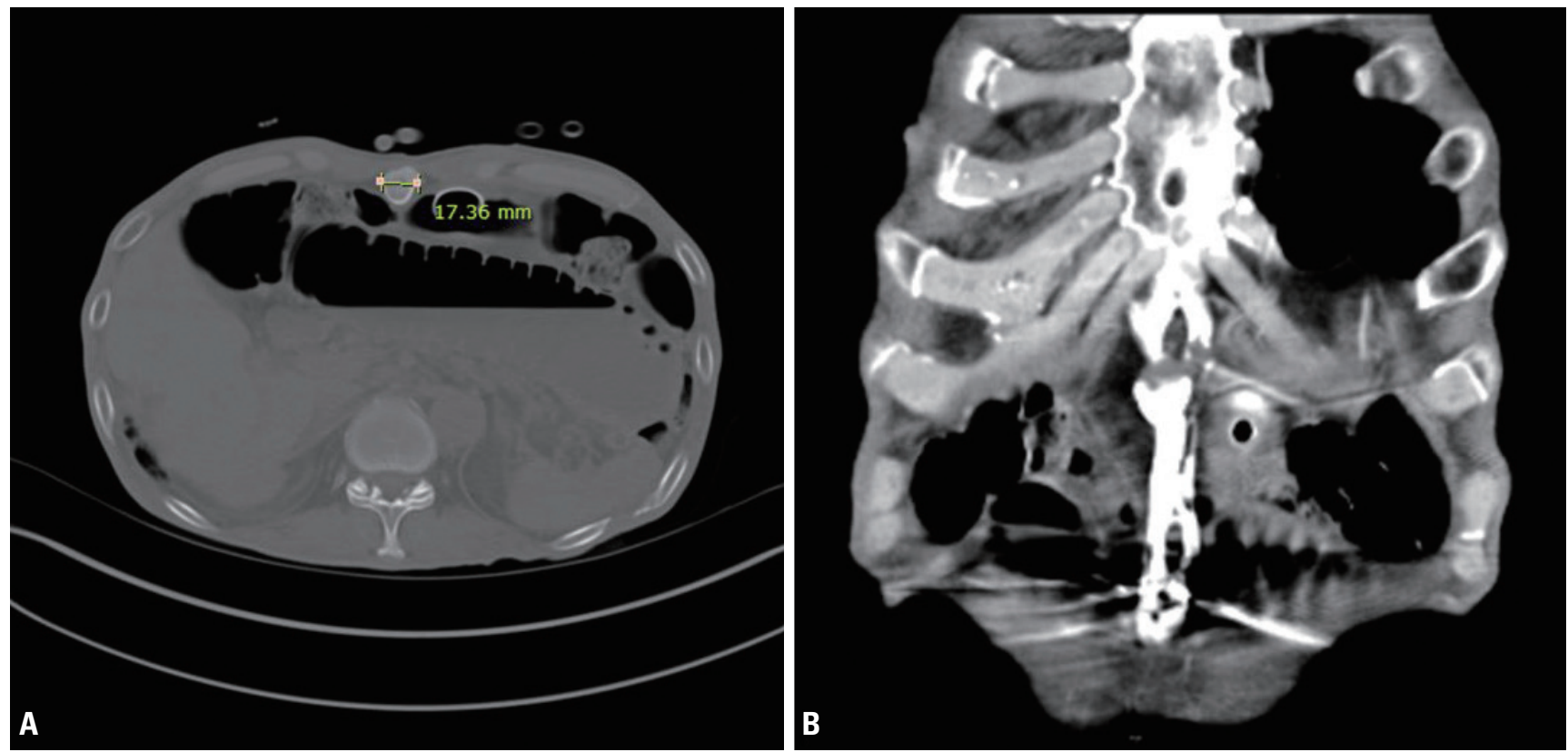

Fig. 2. Follow-up abdominal CT on postoperative 2 years reveals the remnant heterotopic ossification in the abdominal wall (A, B). The diameter of heterotopic bone was $1.7 \mathrm{~cm}$ (A). CT: computed tomography.

ed heterotopic ossification in the abdominal wall with slightly decrease in size $(14 \times 1.7 \mathrm{~cm})$ (Fig. 2). There was no evidence of any other complications.

\section{DISCUSSION}

The incidence of $\mathrm{HO}$ following exploratory laparotomy is unknown. HO is well-documented in the orthopedic literature, with an incidence ranging from $30 \%$ to $60 \%$ following total hip arthroplasty. On the other hand, abdominal heterotopic ossification has been generally considered to be a rare complication. But HO develops in a high proportion of trauma patients following damage control laparotomy. Kim et al. [4] reviewed post-operative CT scans, and identified $\mathrm{HO}$ in $25 \%$ of all patients undergoing open abdominal surgery. Wang et al. [5] report 53\% of patients developed $\mathrm{HO}$ after damage control laparotomy.

It is also difficult to determine the exact time to onset of $\mathrm{HO}$ development. In general, ectopic bone formation in midline incision scars takes place within a few months and almost always within the first year after surgery. In our patient, heterotopic ossification was observed within 2 months of the surgical procedure.
Although many causes have been proposed, the etiology of this pathology is not consensual [1]. Two prevailing theories have emerged regarding the pathogenesis of heterotopic ossification. The first proposes that particles from the periosteum of the xiphoid process or symphysis pubis are inoculated into the wound intra-operatively, leading to bone formation. Others theorize that pluripotent mesenchymal cells can differentiate to osteoblasts in reaction to local injury [6]. While the mechanism remains incompletely understood, both systemic and local factors are thought to participate in creating pro-inflammatory conditions conducive to $\mathrm{HO}$ formation [3].

Although there was substantial variability in the $\mathrm{HO}$ sizes [4], Wang et al. [5] reported that $42 \%$ of all patients developed HO measuring greater than $10 \mathrm{~cm}$ (longitudinal) by $1 \mathrm{~cm}$ (axial). In our case, the size of heterotopic bone was $15 \times 2.3 \mathrm{~cm}$. Although it is symptomatic only in rare cases, it may cause regional abdominal pain and discomfort. Large HOs may be cause substantial patient hardship, related to chronic abdominal pain, discomfort and abdominal wall stiffness [7].

Imaging of ectopic bone formation in abdominal midline incisions appears typical. HOs can be incidental findings on plain radiographs. A lateral plain film will demon- 
strate a calcific or bone density linear structure within the abdominal wall. CT scan can show typical mature peripheral mineralization with central lucency. In our case, CT scan revealed complete ossifications indicative of the mature phase of the pathologic process. Maturation can be examined with activity-oriented imaging as 3-phase bone scan or single-photon emission computed tomography (SPECT) [8].

Radiologically, it is important to distinguish this benign entity from other postoperative complications such as wound infection or foreign bodies and from an intra-incisional primary or metastatic neoplasm. This can be achieved using the typical imaging characteristics of heterotopic bone formation in surgical scars.

The majority of patients with $\mathrm{HO}$ are asymptomatic and don't need any treatment. If symptomatic, treatment consists of complete excision with primary closure $[5,9]$. The main actionable factor in the management of $\mathrm{HO}$ remains prophylaxis. Studies in the orthopedic literature have demonstrated that short-term oral prophylaxis with nonsteroidal anti-inflammatory drugs (NSAIDs) is associated with a decreased incidence of $\mathrm{HO}$ [10]. While the bleeding risks and renal complications associated with NSAIDs are important considerations in the critically ill trauma patient, judicious and timely use of anti-inflammatory drugs may benefit select patients.

The prognosis of HO is generally good, with no evidence of malignant potential so far and treatment should be conservative whenever possible, avoiding surgery to prevent further ossification [1]. Cases with bowel obstruction in symptomatic patients usually require surgical intervention and a complete excision of bone formations should be made.

In conclusion, HOs can be more common than previously suggested and only symptomatic patients need to be treated. This case report is illustrative for HOs after exploratory laparotomy because it concerns a male patient with a midline incision and the location of the ossification in proximity to the xyphoid process. It is an exceptional case because of the size of the $\mathrm{HO}(15 \mathrm{~cm}$ craniocaudal length).

\section{ACKNOWLEDGEMENTS}

This work was supported by clinical research grant from Pusan National University Hospital in 2018.

\section{REFERENCES}

1. Ferreira C, Gomes C, Melo A, Tenreiro N, Pinto B, Moreira $\mathrm{H}$, et al. Heterotopic mesenteric and abdominal wall ossification - two case reports in one institution. Int J Surg Case Rep 2017;37:22-5.

2. Askanazy M, Lubarsch MQ. Zur Kenntniss der Knochenbildungen in Lunge und Pleura. Deutsch Path Gesellsch 1901;3: 102-14.

3. Edwards DS, Kuhn KM, Potter BK, Forsberg JA. Heterotopic ossification: a review of current understanding, treatment, and future. J Orthop Trauma 2016;30 Suppl 3:S27-30.

4. Kim J, Kim Y, Jeong WK, Song SY, Cho OK. Heterotopic ossification developing in surgical incisions of the abdomen: analysis of its incidence and possible factors associated with its development. J Comput Assist Tomogr 2008;32:872-6.

5. Wang Y, Stanek A, Grushka J, Fata P, Beckett A, Khwaja K, et al. Incidence and factors associated with development of heterotopic ossification after damage control laparotomy. Injury 2018;49:51-5.

6. Reardon MJ, Tillou A, Mody DR, Reardon PR. Heterotopic calcification in abdominal wounds. Am J Surg 1997;173:145-7.

7. Suleiman NN, Sandberg LJ. Extensive abdominal wall incisional heterotopic ossification reconstructed with component separation and strattice inlay. Plast Reconstr Surg Glob Open 2016;4:e816.

8. van Leeuwen RJ, Kraal T, Scholtens S, Visser G. A large heterotopic ossification in a 25 years old laparotomy scar. Quant Imaging Med Surg 2016;6:470-3.

9. Ghuman MS, Saggar K. Images in clinical medicine. Heterotopic ossification of a midline abdominal incision. N Engl J Med 2014;371:464.

10. Miller GK. Editorial commentary: the efficacy of nonsteroidal anti-inflammatory drugs for prophylaxis of heterotopic ossification in hip arthroscopy--do we treat patients or X-rays? Arthroscopy 2016;32:526-7. 